TRANSACTIONS OF THE

AMERICAN MATHEMATICAL SOCIETY

Volume 364, Number 5, May 2012, Pages 2647-2666

S 0002-9947(2012)05437-7

Article electronically published on January 19, 2012

\title{
SIGN CHANGES OF THE ERROR TERM IN WEYL'S LAW FOR HEISENBERG MANIFOLDS
}

\author{
KAI-MAN TSANG AND WENGUANG ZHAI
}

\begin{abstract}
Let $R(T)$ be the error term in Weyl's law for the $(2 l+1)$-dimensional Heisenberg manifold $\left(H_{l} / \Gamma, g_{l}\right)$. In this paper, several results on the sign changes and odd moments of $R(t)$ are proved. In particular, it is proved that for some sufficiently large constant $c, R(t)$ changes sign in the interval $[T, T+c \sqrt{T}]$ for all large $T$. Moreover, for a small constant $c_{1}$ there exist infinitely many subintervals in $[T, 2 T]$ of length $c_{1} \sqrt{T} \log ^{-5} T$ such that $\pm R(t)>c_{1} t^{l-1 / 4}$ holds on each of these subintervals.
\end{abstract}

\section{INTRODUCTION}

Let $(M, g)$ be a closed $n$-dimensional Riemannian manifold with metric $g$ and Laplace-Beltrami operator $\Delta$. Let $N(t)$ denote its spectral counting function, which is defined as the number of the eigenvalues of $\Delta$ not exceeding $t$. Hörmander [13] proved that Weyl's law

$$
N(t)=\frac{\operatorname{vol}\left(B_{n}\right) \operatorname{vol}(M)}{(2 \pi)^{n}} t^{n / 2}+O\left(t^{(n-1) / 2}\right)
$$

holds, where $\operatorname{vol}\left(B_{n}\right)$ is the volume of the $n$-dimensional unit ball.

Let

$$
R(t)=N(t)-\frac{\operatorname{vol}\left(B_{n}\right) \operatorname{vol}(M)}{(2 \pi)^{n}} t^{n / 2} .
$$

Hörmander's estimate (1.1) in general is sharp, as the well-known example of the sphere $S^{n}$ with its canonical metric shows [13]. However, it is a very difficult problem to determine the optimal bound of $R(t)$ in any given manifold, which depends on the properties of the associated geodesic flow. Many improvements have been obtained for certain types of manifolds; see 1, 2, 3, 4, 7, 10, 14, 17, 20, 22, 25, 29, 30, 31.

1.1. Weyl's law for $\mathbb{T}^{2}$ : The Gauss circle problem. The simplest compact manifold with integrable geodesic flow is the 2 -torus $\mathbb{T}^{2}=\mathbb{R}^{2} / \mathbb{Z}^{2}$. The exponential functions $e(m x+n y)(m, n \in \mathbb{Z})$ form a basis of eigenfunctions of the Laplace operator $\Delta=\partial_{x}^{2}+\partial_{y}^{2}$, which acts on functions on $\mathbb{T}^{2}$. The corresponding eigenvalues are $4 \pi^{2}\left(m^{2}+n^{2}\right), m, n \in \mathbb{Z}$. The spectral counting function

$$
N_{I}(t)=\left\{\lambda_{j} \in \operatorname{Spec}(\Delta): \lambda_{j} \leq t\right\}
$$

Received by the editors December 5, 2009 and, in revised form, July 24, 2010.

2010 Mathematics Subject Classification. Primary 11N37, 35P20, 58J50.

Key words and phrases. Heisenberg manifold, Weyl's law, error term, Voronoi's formula, sign change.

The work of the second author was supported by National Natural Science Foundation of China (Grant No. 10771127) and Mathematical Tianyuan Foundation (No. 10826028).

(C)2012 American Mathematical Society 
is equal to the number of lattice points of $\mathbb{Z}^{2}$ inside a circle of radius $\sqrt{t} / 2 \pi$. The well-known Gauss circle problem is the study of the properties of the error term of the function $N_{I}(t)$.

In this case, formula (1.1) becomes

$$
N_{I}(t)=\frac{t}{4 \pi}+O\left(t^{1 / 2}\right)
$$

which is the classical result of Gauss. Let $R_{I}(t)$ denote the error term in (1.2). Many authors improved the upper bound estimate of $R_{I}(t)$. The latest result is due to Huxley [14], which reads

$$
R_{I}(t) \ll t^{131 / 416} \log ^{26947 / 8320} t .
$$

Hardy [11] conjectured that

$$
R_{I}(t) \ll t^{1 / 4+\varepsilon}
$$

Cramér [5] proved that

$$
\lim _{T \rightarrow \infty} T^{-3 / 2} \int_{1}^{T}\left|R_{I}(t)\right|^{2} d t=C, \quad C=\frac{1}{6 \pi^{3}} \sum_{n=1}^{\infty} \frac{r^{2}(n)}{n^{3 / 2}},
$$

which is consistent with Hardy's conjecture. Here $r(n)$ denotes the number of ways in which $n$ can be written as a sum of two squares.

Ivić [15] first used the large value technique to study the higher power moments of $R_{I}(t)$. He proved that the estimate

$$
\int_{1}^{T}\left|R_{I}(t)\right|^{A} d t \ll T^{1+A / 4+\varepsilon}
$$

holds for each fixed $0 \leq A \leq 35 / 4$. The value of $A$ for which (1.5) holds is closely related to the upper bound of $R_{I}(t)$. If we insert the estimate (1.3) into Ivić's machinery, we get that (1.5) holds for $0 \leq A \leq 262 / 27$.

The first author [28] studied the third and the fourth moments of $R_{I}(t)$. He proved the following two asymptotic formulas:

$$
\begin{aligned}
& \int_{1}^{T} R_{I}^{3}(t) d t=c_{3} T^{7 / 4}+O\left(T^{7 / 4-1 / 14+\varepsilon}\right) \\
& \int_{1}^{T} R_{I}^{4}(t) d t=c_{4} T^{2}+O\left(T^{2-1 / 23+\varepsilon}\right)
\end{aligned}
$$

where $c_{3}$ and $c_{4}$ are explicit constants.

In [30, the second author proved by a unified method that the asymptotic formula

$$
\int_{1}^{T} R_{I}^{k}(t) d t=c_{k} T^{1+k / 4}+O\left(T^{1+k / 4-\delta_{k}+\varepsilon}\right)
$$

holds for $3 \leq k \leq 9$, where $c_{k}$ and $\delta_{k}>0$ are explicit constants.

1.2. Weyl's law for $(2 l+1)$-dimensional Heisenberg manifold. Let $l \geq 1$ be a fixed integer and $\left(H_{l} / \Gamma, g\right)$ be a $(2 l+1)$-dimensional Heisenberg manifold with a metric $g$. When $l=1$, Petridis and Toth [25] proved that $R(t)=O\left(t^{5 / 6} \log t\right)$ for a special metric. Later in [4] this bound was improved to $O\left(t^{119 / 146+\varepsilon}\right)$ for all left-invariant Heisenberg metrics. For $l>1$ Khosravi and Petridis [20] proved that $R(t)=O\left(t^{l-7 / 41}\right)$ holds for rational Heisenberg manifolds. In both [4] and 20] they 
first established a $\psi$-expression of $R(t)$ and then used the van der Corput method of exponential sums. Substituting Huxley's result of [14] into the arguments of [4] and 20], we can get that the estimate

$$
R(t)=O\left(t^{l-77 / 416}(\log t)^{26947 / 8320}\right)
$$

holds for all rational $(2 l+1)$-dimensional Heisenberg manifolds.

It was conjectured that for rational Heisenberg manifolds, the pointwise estimate

$$
R(t) \ll t^{l-1 / 4+\varepsilon}
$$

holds, which was proposed in Petridis and Toth 25 for the case $l=1$ and in Khosravi and Petridis 20] for the case $l>1$. As an evidence of this conjecture, Petridis and Toth proved the following $L^{2}$ result:

$$
\int_{I^{3}}\left|N(t ; \vec{u})-\frac{1}{6 \pi^{2}} \operatorname{vol}(M(\vec{u})) t^{3 / 2}\right|^{2} d \vec{u} \leq C_{\delta} t^{3 / 2+\delta}
$$

for the 3-dimensional Heisenberg manifold $H_{1}$, where $N(t ; \vec{u})$ is the counting function for $H_{1}$ with the metric

$$
g(\vec{u})=\left(\begin{array}{llc}
u_{1}^{-1} & 0 & 0 \\
0 & u_{2}^{-1} & 0 \\
0 & 0 & u_{3}^{-1}
\end{array}\right)
$$

for any $\vec{u}=\left(u_{1}, u_{2}, u_{3}\right) \in I^{3}$, and $I=[1-\varepsilon, 1+\varepsilon]$. They also proved

$$
\frac{1}{T} \int_{T}^{2 T}\left|N(t)-\frac{1}{6 \pi^{2}} \operatorname{vol}(M) t^{3 / 2}\right| d t \gg T^{3 / 4} .
$$

Now let $M=\left(H_{l} / \Gamma, g_{l}\right)$ be a $(2 l+1)$-dimensional Heisenberg manifold with the metric

$$
g_{l}:=\left(\begin{array}{ll}
I_{2 l \times 2 l} & 0 \\
0 & 2 \pi
\end{array}\right),
$$

where $I_{2 l \times 2 l}$ is the identity matrix.

Khosravi and Toth [21] proved that

$$
\int_{1}^{T} R(t)^{2} d t=C_{2, l} T^{2 l+1 / 2}+O\left(T^{2 l+1 / 4+\varepsilon}\right),
$$

where $C_{2, l}$ is an explicit constant.

Khosravi [19] proved that the asymptotic formula

$$
\int_{1}^{T} R^{3}(t) d t=C_{3, l} T^{3 l+1 / 4}+O\left(T^{3 l+3 / 14+\varepsilon}\right)
$$

is true for some explicit constant $C_{3, l}$.

In [32] the second author proved that the asymptotic formula

$$
\int_{1}^{T} R^{k}(t) d t=C_{k, l} T^{k(l-1 / 4)+1}+O\left(T^{k(l-1 / 4)+1-\eta_{k}+\varepsilon}\right)
$$

holds true for any $3 \leq k \leq 9$, where $C_{k, l}$ and $\eta_{k}>0$ are explicit constants.

Recently, Nowak [23, 24] proved that the estimate

$$
\limsup _{t \rightarrow \infty} \frac{R(t)}{t^{l-1 / 4} \omega_{l}(t)}>0
$$


holds with

$$
\omega_{l}(t)= \begin{cases}(\log t)^{1 / 4}, & \text { if } l \text { is even, } \\ \left(\log _{2} t \log _{3} t\right)^{1 / 4}, & \text { if } l \text { is odd, }\end{cases}
$$

where $\log _{r} t=\log \log _{r-1} t, \log _{1} t=\log t$.

Notation. For a real number $t$, let $[t]$ denote the integer part of $t,\{t\}=t-[t],\|t\|=$ $\min (\{t\}, 1-\{t\}), e(t)=e^{2 \pi i t}$. $\varepsilon$ always denotes a sufficiently small positive constant. $\mathbb{R}, \mathbb{Z}, \mathbb{N}$ denote the set of real numbers, the set of integers, and the set of positive integers, respectively. $d(n)$ denotes the Dirichlet divisor function. Throughout this paper, $\mathcal{L}$ always denotes $\log T$.

\section{Sign CHANGES of $R(t)$}

From now on, we always suppose that $R(t)$ denotes the error term in Weyl's law for the $(2 l+1)$-dimensional Heisenberg manifold $\left(H_{l} / \Gamma, g_{l}\right)$.

In [12, Heath-Brown and the first author studied the sign changes of the error term $R_{I}(t)$. They proved that for a suitable constant $C>0, R_{I}(t)$ changes sign on the interval $[T, T+C \sqrt{T}]$ for every sufficiently large $T$. Here the length $\sqrt{T}$ is almost best possible since they proved that in the interval $[T, 2 T]$ there are many subintervals of length $\gg \sqrt{T} \log ^{-5} T$ such that $R_{I}(t)$ does not change sign in any of these subintervals.

In this paper we shall show that similar results hold for $R(t)$. More precisely, we have the following theorems.

Theorem 1. Let $c_{1}>0$ be a sufficiently small constant and $c_{2}>0$ be a sufficiently large constant. For any real-valued function $g(t)$ satisfying $|g(t)| \leq c_{1} t^{l-1 / 4}$, the function $R(t)+g(t)$ changes sign at least once in the interval $\left[T, T+c_{2} \sqrt{T}\right]$ for

every sufficiently large $T$. In particular, there exist $t_{1}, t_{2} \in\left[T, T+c_{2} \sqrt{T}\right]$ such that $R\left(t_{1}\right) \geq c_{1} t_{1}^{l-1 / 4}$ and $R\left(t_{2}\right) \leq-c_{1} t_{2}^{l-1 / 4}$.

Theorem 2. There exist three positive absolute constants $c_{3}, c_{4}, c_{5}$ such that, for any large parameter $T$, there are at least $c_{3} \sqrt{T} \log ^{5} T$ disjoint subintervals of length $c_{4} \sqrt{T} \log ^{-5} T$ in $[T, 2 T]$ such that $\pm R(t)>c_{5} t^{l-1 / 4}$ whenever $t$ lies in any of these subintervals. We also have the estimate

$$
\text { meas }\left\{t \in[T, 2 T]: \pm R(t)>c_{5} t^{l-1 / 4}\right\} \gg T .
$$

Remark 1. Our proof of Theorem 2 is a variant of the proof of Theorem 2 in Section 3 of [12. However, our approach can prove that $R(t)$ (respectively $-R(t)$ ) has large values on long intervals of length $\gg \sqrt{T} \log ^{-5} T$.

As an application of Theorem 2, we study the $\Omega$-result of the error term in the asymptotic formula (1.13) for odd $k$. For any integer $k \geq 2$, define

$$
\mathcal{F}_{k, l}(T):=\int_{1}^{T} R^{k}(t) d t-C_{k, l} T^{k(l-1 / 4)+1} .
$$

We then have the following

Theorem 3. The estimate

$$
\mathcal{F}_{k, l}(T)=\Omega\left(T^{k(l-1 / 4)+1 / 2} \log ^{-5} T\right)
$$

holds for any fixed odd integer $k \geq 3$. 
Remark 2. The results of [32] show that (1.13) should be true for any integer $k \geq 3$. However, up to the present we can only prove it for $3 \leq k \leq 9$. Theorem 3 provides an $\Omega$-result for any odd $k \geq 3$.

The corresponding result on $R_{I}(t)$ proved in [12] can be improved slightly via the same approach. We state it as the following theorem.

Theorem 4. There exist three positive absolute constants $c_{6}, c_{7}, c_{8}$ such that, for any large parameter $T$, there are at least $c_{6} \sqrt{T} \log ^{3} T$ disjoint subintervals of length $c_{7} \sqrt{T} \log ^{-3} T$ in $[T, 2 T]$ such that $\pm R_{I}(t)>c_{8} t^{1 / 4}$ whenever $t$ lies in any of these subintervals. We also have the estimate

$$
\operatorname{meas}\left\{t \in[T, 2 T]: \pm R_{I}(t)>c_{8} t^{1 / 4}\right\} \gg T \text {. }
$$

Remark 3. By Theorem 4, the argument of Theorem 3 proves that the formula

$$
\int_{1}^{T} R_{I}^{k}(t) d t=c_{k} T^{1+k / 4}+\Omega\left(T^{(k+2) / 4} \log ^{-3} T\right)
$$

holds for any odd integer $k \geq 3$.

For the error term $\Delta(x)$ in the divisor problem, the asymptotic formula (see [28] and [30])

$$
\int_{1}^{T} \Delta^{k}(x) d x=C_{k} T^{k / 4+1}+O\left(T^{k / 4+1-\eta_{k}}\right)
$$

holds for any integer $3 \leq k \leq 9$, where $C_{k}$ and $\eta_{k}$ are explicit constants. In [16], Ivić and the second author proved the estimate

$$
\int_{1}^{T} \Delta^{k}(x) d x-C_{k} T^{k / 4+1}=\Omega\left(G^{k+1}(T) \log ^{-1} T\right)
$$

for any $k \geq 2$, where

$$
G(x)=(x \log x)^{1 / 4}(\log \log x)^{\frac{3}{4}\left(2^{4 / 3}-1\right)}(\log \log \log x)^{-5 / 8}
$$

is the $\Omega$-estimate of $\Delta(x)$ proved by Soundararajan [26]. In view of the work in 12, the proof of Theorem 3 implies that, for any odd integer $k \geq 3$, the estimate $\Omega\left(G^{k+1}(T) \log ^{-1} T\right)$ can be substantially improved to $\Omega\left(T^{(k+2) / 4} \log ^{-5} T\right)$. A similar result also holds for $E(t)$, the error term in the mean square of the Riemann zeta-function $\zeta(s)$ over the critical line.

\section{Background of Heisenberg manifolds AND THE ANALOGUE VORONOI FORMUla FOR $R(2 \pi x)$}

In this section, we first review some background of Heisenberg manifolds. The reader can refer to [6], [9, 27] for more details.

3.1. Heisenberg manifolds. Suppose $x \in \mathbb{R}^{l}$ is a row vector and $y \in \mathbb{R}^{l}$ is a column vector. Define

$$
\gamma(x, y, t)=\left(\begin{array}{lll}
1 & x & t \\
0 & I_{l} & y \\
0 & 0 & 1
\end{array}\right), \quad X(x, y, t)=\left(\begin{array}{ccc}
0 & x & t \\
0 & 0 & y \\
0 & 0 & 0
\end{array}\right) .
$$

The $(2 l+1)$-dimensional Heisenberg group $H_{l}$ is defined by

$$
H_{l}=\left\{\gamma(x, y, t): x, y \in \mathbb{R}^{l}, t \in \mathbb{R}\right\},
$$


and its Lie algebra is

$$
\mathfrak{H}_{l}=\left\{X(x, y, t): x, y \in \mathbb{R}^{l}, t \in \mathbb{R}\right\} .
$$

We say $\Gamma$ is a uniform discrete subgroup of $H_{l}$ if $H_{l} / \Gamma$ is compact. A $(2 l+1)$ dimensional Heisenberg manifold is a pair $\left(H_{l} / \Gamma, g\right)$ for which $\Gamma$ is a uniform discrete subgroup of $H_{l}$ and $g$ is a left $H_{l}$-invariant metric.

For every $l$-tuple $r=\left(r_{1}, r_{2}, \cdots, r_{l}\right) \in \mathbb{N}^{l}$ such that $r_{j} \mid r_{j+1}(j=1,2, \cdots, l-1)$, let $r \mathbb{Z}^{l}$ denote the $l$-tuple $x=\left(x_{1}, x_{2}, \cdots, x_{l}\right)$ with $x_{j} \in r_{j} \mathbb{Z}$. Define

$$
\Gamma_{r}=\left\{\gamma(x, y, t): x \in r \mathbb{Z}^{l}, y \in r \mathbb{Z}^{l}, t \in \mathbb{Z}\right\} .
$$

It is clear that $\Gamma_{r}$ is a uniform discrete subgroup of $H_{l}$. According to Theorem 2.4 of [9], the subgroup $\Gamma_{r}$ classifies all the uniform discrete subgroups of $H_{l}$ up to automorphisms. Thus (see 9], Corollary 2.5) given any Riemannian Heisenberg manifold $M=\left(H_{l} / \Gamma, g\right)$, there exists a unique $l$-tuple $r$ as before and a left-invariant metric $\tilde{g}$ on $H_{l}$ such that $M$ is isometric to $\left(H_{l} / \Gamma, \tilde{g}\right)$. So (see [9, 2.6(b)) we can replace the metric $g$ by $\phi^{*} g$, where $\phi$ is an inner automorphism such that the direct sum split of the Lie algebra $\mathfrak{H}_{l}=\mathbb{R}^{2 l} \oplus \mathfrak{Z}$ is orthogonal . Here $\mathfrak{Z}$ is the center of the Lie algebra and

$$
\mathbb{R}^{2 l}=\left\{\left(\begin{array}{lll}
0 & x & 0 \\
0 & 0 & y \\
0 & 0 & 0
\end{array}\right): x, y \in \mathbb{R}^{l}\right\} .
$$

With respect to this orthogonal split of $H_{l}$ the metric $g$ has the form

$$
\left(\begin{array}{ll}
h & 0 \\
0 & g_{2 l+1}
\end{array}\right) \text {, }
$$

where $h$ is a positive-definite $2 l \times 2 l$ matrix and $g_{2 l+1}>0$ is a real number.

The volume of the Heisenberg manifold is given by

$$
\operatorname{vol}\left(H_{l} / \Gamma, g\right)=\left|\Gamma_{r}\right| \sqrt{\operatorname{det}(g)}
$$

with $\left|\Gamma_{r}\right|=r_{1} r_{2} \cdots r_{l}$ for $r=\left(r_{1}, r_{2}, \cdots, r_{l}\right)$.

3.2. The spectrum of Heisenberg manifolds. Let $\Sigma$ be the spectrum of the Laplacian on $M=\left(H_{l} / \Gamma, g_{l}\right)$, where the eigenvalues are counted with multiplicities. According to 9 (p. 258), $\Sigma$ can be divided into two parts, $\Sigma_{1}$ and $\Sigma_{2}$, where $\Sigma_{1}$ is the spectrum of $2 l$-dimensional torus and $\Sigma_{2}$ contains all eigenvalues of the form

$$
2 \pi m^{2}+2 \pi m\left(2 n_{1}+\cdots+2 n_{l}+l\right), m \in \mathbb{N}, n_{j} \in \mathbb{N} \cup\{0\},
$$

each eigenvalue counted with the multiplicity $2 m^{l}$.

3.3. The Voronoi-type formula for $R(2 \pi x)$. In 32 the second author proved an analogue Voronoi formula for $R^{*}(x):=R(2 \pi x)$. Suppose $T \geq 10$ is a large parameter, $\mathcal{L}=\log T$. Suppose $T \leq x \leq 2 T, H \geq T$, and $J=[(\mathcal{L}-\log \mathcal{L}) / 2 \log 2]$. Then we have

$$
\begin{gathered}
R^{*}(x)=\frac{2^{2-l} x^{l-1 / 4}}{(l-1) ! \pi} \sum_{\substack{1 \leq n \leq H^{2}\left(2^{2 J+1}+1 / 2\right) \\
+}} \frac{\tau_{l}(n ; H, T)}{n^{3 / 4}} \cos \left(2 \pi \sqrt{x n}-\frac{\pi}{4}\right) \\
+O\left(T^{l-1 / 2} G(x)+T^{l-1 / 2} \mathcal{L}^{2}\right),
\end{gathered}
$$


where

$$
\begin{aligned}
\tau_{l}(n ; H, T): \quad & \sum_{\substack{n=h(2 r-h), 1 \leq h \leq H \\
h \leq r \leq h\left(2^{2 J+1}+1 / 2\right)}} \frac{e(l h / 2) h^{1 / 2}}{(2 r-h)^{1 / 2}}\left(1-\frac{h}{2 r-h}\right)^{l-1}, \\
G(x) & =\sum_{m \leq \sqrt{2 T}} \min \left(1, \frac{1}{H\left\|\frac{x}{2 m}-\frac{m}{2}+\frac{l}{2}\right\|}\right) .
\end{aligned}
$$

We note that if $n \leq T \mathcal{L}^{-1}$, then

$$
\tau_{l}(n ; H, T)=\tau_{l}(n):=\sum_{\substack{n=h(2 r-h) \\ h \leq r}} \frac{e(l h / 2) h^{1 / 2}}{(2 r-h)^{1 / 2}}\left(1-\frac{h}{2 r-h}\right)^{l-1} .
$$

Remark 3.1. There is an error in the definition of $\tau_{l}(n)$ in [32, where the important condition $h \leq r$ was omitted.

Remark 3.2. The term $T^{l-1 / 2} \mathcal{L}^{2}$ in (3.1) reads as $T^{l-1 / 2} \mathcal{L}^{3}$ in Proposition 6.1 of 32. However, by a little more analysis in Section 6.2 of [32, we see that $T^{l-1 / 2} \mathcal{L}^{3}$ can be replaced by $T^{l-1 / 2} \mathcal{L}^{2}$.

\section{Proof of Theorem 1}

In this section we prove Theorem 1 . We follow the approach of 12 .

Let $n_{0}$ denote the smallest integer $n$ such that $\tau_{l}(n) \neq 0$. From the definition of $\tau_{l}(n)$ it is easy to see that $n_{0}=1$ if $l=1$ and $n_{0}=3$ if $l>1$, and indeed

$$
\tau_{l}\left(n_{0}\right)= \begin{cases}-1, & \text { if } l=1 \\ e(l / 2) 3^{1 / 2-l} 2^{l-1}, & \text { if } l>1\end{cases}
$$

Suppose $|g(t)| \leq c_{1} t^{l-1 / 4}$. Let

$$
R^{* *}(t)=t^{-(2 l-1 / 2)}\left(R\left(2 \pi t^{2}\right)+g\left(2 \pi t^{2}\right)\right), \quad t \geq 1,
$$

and define

$$
K_{\zeta}(u):=(1-|u|)\left(1+\zeta \sin 2 \pi \alpha \sqrt{n_{0}} u\right), \quad u \geq 1,
$$

where $\zeta=1$ or -1 and $\alpha>2$ is a large constant.

It is easy to see that Theorem 1 follows from Lemma 4.1 below.

Lemma 4.1. Suppose $T \geq 10$ is a large parameter. Then for each $\sqrt{T} \leq t \leq \sqrt{2 T}$, we have

$$
\begin{gathered}
\int_{-1}^{1} R^{* *}(t+\alpha u) K_{\zeta}(u) d u=-\frac{\zeta 2^{1-l} \tau_{l}\left(n_{0}\right)}{(l-1) ! \pi n_{0}^{3 / 4}} \sin \left(2 \pi \sqrt{n_{0}} t-\frac{\pi}{4}\right)+O\left(\alpha^{-1}\right) \\
+O\left(t^{-(2 l-1 / 2)} \sup _{|u| \leq 1}\left|g\left(2 \pi(t+\alpha u)^{2}\right)\right|+t^{-1 / 2} \log ^{2} t\right) .
\end{gathered}
$$


Proof. From (3.1) and the definition of $n_{0}$ we have

$$
\begin{aligned}
t^{-(2 l-1 / 2)} R^{*}\left(t^{2}\right) & =\frac{2^{2-l}}{(l-1) ! \pi} \sum_{n_{0} \leq n \leq H^{2}\left(2^{2 J+1}+1 / 2\right)} \frac{\tau_{l}(n ; H, T)}{n^{3 / 4}} \\
& \times \cos \left(2 \pi t \sqrt{n}-\frac{\pi}{4}\right)+O\left(t^{-1 / 2} G_{1}(t)+t^{-1 / 2} \log ^{2} t\right), \\
G_{1}(t) & =\sum_{m \leq \sqrt{2 T}} \min \left(1, \frac{1}{H\left\|\frac{t^{2}}{2 m}-\frac{m}{2}+\frac{l}{2}\right\|}\right) .
\end{aligned}
$$

We first estimate the integral $\int_{-1}^{1} G_{1}(t+\alpha u) d u$. It is well known that

$$
\min \left(1, \frac{1}{H\|r\|}\right)=\sum_{h=-\infty}^{\infty} a(h) e(h r)
$$

with

Thus we have

$$
a(0) \ll \frac{\log H}{H}, \quad a(h) \ll \min \left(\frac{\log H}{H}, \frac{H}{h^{2}}\right), h \neq 0 .
$$

$$
\begin{aligned}
\int_{-1}^{1} G_{1}(t+\alpha u) d u & =\sum_{h=-\infty}^{\infty} a(h) \sum_{m \leq \sqrt{2 T}} e\left(\frac{h t^{2}}{2 m}-\frac{h m}{2}+\frac{h l}{2}\right) \\
& \times \int_{-1}^{1} e\left(\frac{2 h t \alpha u+h \alpha^{2} u^{2}}{2 m}\right) d u \\
& \ll \sqrt{T}|a(0)|+\sum_{h=1}^{\infty}|a(h)| \sum_{m \leq \sqrt{2 T}} \frac{m}{h t \alpha} \\
& \ll \sqrt{T} H^{-1} \log ^{2} H,
\end{aligned}
$$

where the first derivative test was used.

Let

$$
J_{\zeta}(\alpha, t, n):=\int_{-1}^{1} \cos \left(2 \pi(t+\alpha u) \sqrt{n}-\frac{\pi}{4}\right) K_{\zeta}(u) d u .
$$

Then we have

$$
J_{\zeta}(\alpha, t, n)=J_{1}-J_{2}+J_{3}-J_{4},
$$

where

$$
\begin{aligned}
& J_{1}=\cos \left(2 \pi t \sqrt{n}-\frac{\pi}{4}\right) \int_{-1}^{1}(1-|u|) \cos (2 \pi \alpha u \sqrt{n}) d u, \\
& J_{2}=\sin \left(2 \pi t \sqrt{n}-\frac{\pi}{4}\right) \int_{-1}^{1}(1-|u|) \sin (2 \pi \alpha u \sqrt{n}) d u \\
& J_{3}=\zeta \cos \left(2 \pi t \sqrt{n}-\frac{\pi}{4}\right) \int_{-1}^{1}(1-|u|) \cos (2 \pi \alpha u \sqrt{n}) \sin \left(2 \pi \alpha \sqrt{n_{0}} u\right) d u \\
& J_{4}=\zeta \sin \left(2 \pi t \sqrt{n}-\frac{\pi}{4}\right) \int_{-1}^{1}(1-|u|) \sin (2 \pi \alpha u \sqrt{n}) \sin \left(2 \pi \alpha \sqrt{n_{0}} u\right) d u .
\end{aligned}
$$

It is easy to see that $J_{2}=J_{3}=0$. By the first derivative test we get that

$$
J_{1} \ll \alpha^{-1} n^{-1 / 2} \text {. }
$$


For $J_{4}$ we have

$$
\begin{aligned}
J_{4}=\frac{\zeta}{2} \sin ( & \left.2 \pi t \sqrt{n}-\frac{\pi}{4}\right) \int_{-1}^{1}(1-|u|) \\
& \times\left(\cos \left(2 \pi \alpha\left(\sqrt{n}-\sqrt{n_{0}}\right) u\right)-\cos \left(2 \pi \alpha\left(\sqrt{n}+\sqrt{n_{0}}\right) u\right)\right) d u .
\end{aligned}
$$

So by the first derivative test again we get

$$
J_{4}= \begin{cases}-\frac{\zeta}{2} \sin \left(2 \pi \sqrt{n_{0}} t-\frac{\pi}{4}\right)+O\left(\alpha^{-1}\right), & \text { if } n=n_{0}, \\ \ll \alpha^{-1} n^{-1 / 2}, & \text { if } n \neq n_{0},\end{cases}
$$

which combining (4.8) and (4.9) gives

$$
J_{\zeta}(\alpha, t, n)= \begin{cases}-\frac{\zeta}{2} \sin \left(2 \pi \sqrt{n_{0}} t-\frac{\pi}{4}\right)+O\left(\alpha^{-1}\right), & \text { if } n=n_{0}, \\ \ll \alpha^{-1} n^{-1 / 2}, & \text { if } n \neq n_{0} .\end{cases}
$$

From (4.4), (4.5), (4.7) and (4.10) we get (taking $H=T^{2}$ )

$$
\begin{aligned}
& \int_{-1}^{1} R^{* *}(t+\alpha u) K_{\zeta}(u) d u \\
= & \frac{2^{2-l}}{(l-1) ! \pi} \sum_{n_{0} \leq n \leq H^{2}\left(2^{2 J+1}+1 / 2\right)} \frac{\tau_{l}(n ; H, T)}{n^{3 / 4}} J_{\zeta}(\alpha, t, n) \\
& +O\left(t^{-(2 l-1 / 2)} \sup _{|u| \leq 1}\left|g\left(2 \pi(t+\alpha u)^{2}\right)\right|+T^{1 / 2} H^{-1} \mathcal{L}^{2}+t^{-1 / 2} \log ^{2} t\right) \\
= & -\frac{\zeta 2^{1-l} \tau_{l}\left(n_{0}\right)}{(l-1) ! \pi n_{0}^{3 / 4}} \sin \left(2 \pi \sqrt{n_{0}} t-\frac{\pi}{4}\right)+O\left(\alpha^{-1}\right)+\sum_{n_{0}+1 \leq n \leq H^{2}\left(2^{2 J+1}+1 / 2\right)} \frac{\left|\tau_{l}(n)\right|}{\alpha n^{5 / 4}} \\
& +O\left(t^{-(2 l-1 / 2)} \sup _{|u| \leq 1}\left|g\left(2 \pi(t+\alpha u)^{2}\right)\right|+t^{-1 / 2} \log ^{2} t\right) \\
= & -\frac{\zeta 2^{1-l} \tau_{l}\left(n_{0}\right)}{(l-1) ! \pi n_{0}^{3 / 4}} \sin \left(2 \pi \sqrt{n_{0}} t-\frac{\pi}{4}\right)+O\left(\alpha^{-1}\right) \\
& +O\left(t^{-(2 l-1 / 2)} \sup _{|u| \leq 1}\left|g\left(2 \pi(t+\alpha u)^{2}\right)\right|+t^{-1 / 2} \log ^{2} t\right)
\end{aligned}
$$

\section{The mean value of $R(t)$ in Short Intervals}

Suppose $T \geq 10$ is a large parameter, $1 \leq h \leq \frac{1}{2} \sqrt{T}$. In this section we shall estimate the integral

$$
I(T, h)=\int_{1}^{T}(R(x+h)-R(x))^{2} d x
$$

which would play an important role in the proof of Theorem 2 . This type of integral was studied for the error term in the mean square of $\zeta(1 / 2+i t)$ by Good [8] and for the error term in the Dirichlet divisor problem by Jutila [18. Our approach is based on Jutila [18, but with some modifications.

Without loss of generality, we shall estimate the integral

$$
I^{*}(T, h)=\int_{1}^{T}\left(R^{*}(x+h)-R^{*}(x)\right)^{2} d x,
$$


where $R^{*}(x)$ was defined in (3.1). We shall prove the following

Lemma 5.1. The estimate

$$
I^{*}(T, h) \ll T^{2 l} h \log ^{3} \frac{\sqrt{T}}{h}+T^{2 l} \mathcal{L}^{4}
$$

holds uniformly for $1 \leq h \leq \frac{1}{2} \sqrt{T}$.

Remark. Lemma 5.1 is also true for $I(T, h)$.

Proof. Write

$$
I^{*}(T, h)=\int_{1}+\int_{2}
$$

where

$$
\begin{aligned}
& \int_{1}:=\int_{1}^{100 \max \left(h^{2}, T^{2 / 3}\right)}\left(R^{*}(x+h)-R^{*}(x)\right)^{2} d x, \\
& \int_{2}:=\int_{100 \max \left(h^{2}, T^{2 / 3}\right)}^{T}\left(R^{*}(x+h)-R^{*}(x)\right)^{2} d x .
\end{aligned}
$$

From (1.11) we have

$$
\int_{1} \ll h^{2(2 l+1 / 2)}+T^{\frac{2}{3}(2 l+1 / 2)} \ll T^{2 l} h .
$$

In order to bound $\int_{2}$, we first estimate the integral

$$
J(U, h)=\int_{U}^{2 U}\left(R^{*}(x+h)-R^{*}(x)\right)^{2} d x, \quad 100 \max \left(h^{2}, T^{2 / 3}\right) \leq U \leq T .
$$

In (3.1) we use $U$ in place of $T$ and then take $H=U^{100}, J=[(\log U-$ $\log \log U) / 2 \log 2]$. Let $z:=\min \left(\varepsilon U h^{-1}, U \log ^{-1} U\right)$. Define

$$
\begin{aligned}
& R_{1}(x):=\frac{2^{2-l} x^{l-1 / 4}}{(l-1) ! \pi} \sum_{1 \leq n \leq z} \frac{\tau_{l}(n)}{n^{3 / 4}} \cos \left(2 \pi \sqrt{n x}-\frac{\pi}{4}\right) \\
& R_{2}(x):=\frac{2^{2-l} x^{l-1 / 4}}{(l-1) ! \pi} \sum_{z \leq n \leq H^{2}\left(2^{2 J+1}+1 / 2\right)} \frac{\tau_{l}(n ; H, T)}{n^{3 / 4}} \cos \left(2 \pi \sqrt{n x}-\frac{\pi}{4}\right) .
\end{aligned}
$$

Then we have

$$
R^{*}(x)=R_{1}(x)+R_{2}(x)+O\left(U^{l-1 / 2} G_{2}(x)+U^{l-1 / 2} \log ^{2} U\right),
$$

where

$$
G_{2}(x):=\sum_{m \leq \sqrt{2 U}} \min \left(1, \frac{1}{H\left\|\frac{x}{2 m}-\frac{m}{2}+\frac{l}{2}\right\|}\right) .
$$

From (6.30) of 32 we have

$$
\int_{U}^{2 U}\left|R_{2}(x)\right|^{2} d x \ll U^{2 l+1 / 2} z^{-1 / 2} \log ^{3} z
$$

Lemma 6.1 of 32 implies that (trivially $G_{2}(x) \ll \sqrt{U}$ )

$$
\int_{U}^{2 U}\left|U^{l-1 / 2} G_{2}(x)\right|^{2} d x \ll U^{2 l-1 / 2} \int_{U}^{2 U} G_{2}(x) d x \ll U^{2 l-99} \log H .
$$


Let

$$
M(x)=R_{2}(x)+O\left(U^{l-1 / 2} G_{2}(x)+U^{l-1 / 2} \log ^{2} U\right) .
$$

Then (5.6) and (5.7) implies

$$
\begin{aligned}
\int_{U}^{2 U}|M(x)|^{2} d x & \ll U^{2 l+1 / 2} z^{-1 / 2} \log ^{3} z+U^{2 l} \log ^{4} U \\
& \ll h^{1 / 2} U^{2 l} \log ^{3} z+U^{2 l} \log ^{4} U .
\end{aligned}
$$

Now we estimate $\int_{U}^{2 U}\left(R_{1}(x+h)-R_{1}(x)\right)^{2} d x$. From the definition of $R_{1}(x)$ we have

$$
R_{1}(x+h)-R_{1}(x)=F_{1}(x)+F_{2}(x),
$$

where

$$
\begin{aligned}
F_{1}(x)= & \frac{2^{2-l}}{(l-1) ! \pi}\left((x+h)^{l-1 / 4}-x^{l-1 / 4}\right) \sum_{1 \leq n \leq z} \frac{\tau_{l}(n)}{n^{3 / 4}} \\
& \quad \times \cos \left(2 \pi \sqrt{n(x+h)}-\frac{\pi}{4}\right) \ll h x^{-1}\left|R_{1}(x+h)\right|, \\
F_{2}(x)= & \frac{2^{2-l}}{(l-1) ! \pi} x^{l-1 / 4} \sum_{1 \leq n \leq z} \frac{\tau_{l}(n)}{n^{3 / 4}} \\
\times & \left(\cos \left(2 \pi \sqrt{n(x+h)}-\frac{\pi}{4}\right)-\cos \left(2 \pi \sqrt{n x}-\frac{\pi}{4}\right)\right) .
\end{aligned}
$$

For the mean square of $F_{1}(x)$ we have

$$
\int_{U}^{2 U}\left|F_{1}(x)\right|^{2} d x \ll h^{2} U^{-2} U^{2 l+1 / 2} \ll h U^{2 l} .
$$

We write

$$
F_{2}^{2}(x)=F_{21}(x)+F_{22}(x)
$$

where

$$
\begin{aligned}
F_{21}(x) & =\frac{2^{4-2 l}}{(l-1) !^{2} \pi^{2}} x^{2 l-1 / 2} \sum_{1 \leq n \leq z} \frac{\tau_{l}^{2}(n)}{n^{3 / 2}} \\
& \times\left(\cos \left(2 \pi \sqrt{n(x+h)}-\frac{\pi}{4}\right)-\cos \left(2 \pi \sqrt{n x}-\frac{\pi}{4}\right)\right)^{2}, \\
F_{22}(x) & =\frac{2^{4-2 l}}{(l-1) !^{2} \pi^{2}} x^{2 l-1 / 2} \sum_{1 \leq m \neq n \leq z} \frac{\tau_{l}(m) \tau_{l}(n)}{(m n)^{3 / 4}} \\
& \times\left(\cos \left(2 \pi \sqrt{m(x+h)}-\frac{\pi}{4}\right)-\cos \left(2 \pi \sqrt{m x}-\frac{\pi}{4}\right)\right) \\
& \times\left(\cos \left(2 \pi \sqrt{n(x+h)}-\frac{\pi}{4}\right)-\cos \left(2 \pi \sqrt{n x}-\frac{\pi}{4}\right)\right) .
\end{aligned}
$$

By writing

$$
\begin{gathered}
\cos \left(2 \pi \sqrt{n(x+h)}-\frac{\pi}{4}\right)-\cos \left(2 \pi \sqrt{n x}-\frac{\pi}{4}\right) \\
=\sum_{j=0}^{1}(-1)^{j+1} \cos \left(2 \pi \sqrt{n(x+j h)}-\frac{\pi}{4}\right)
\end{gathered}
$$


we get

$$
\begin{aligned}
F_{22}(x)= & \frac{2^{4-2 l} x^{2 l-1 / 2}}{(l-1) !^{2} \pi^{2}} \sum_{j_{1}=0}^{1} \sum_{j_{2}=0}^{1}(-1)^{j_{1}+j_{2}} \sum_{1 \leq m \neq n \leq z} \frac{\tau_{l}(m) \tau_{l}(n)}{(m n)^{3 / 4}} \\
& \times \cos \left(2 \pi \sqrt{m\left(x+j_{1} h\right)}-\frac{\pi}{4}\right) \times \cos \left(2 \pi \sqrt{n\left(x+j_{2} h\right)}-\frac{\pi}{4}\right) .
\end{aligned}
$$

By the elementary formula

$$
\cos a \cos b=\frac{\cos (a-b)+\cos (a+b)}{2}
$$

we have

$$
F_{22}(x)=F_{221}(x)+F_{222}(x),
$$

where

$$
\begin{aligned}
& F_{221}(x)=\frac{2^{3-2 l} x^{2 l-1 / 2}}{(l-1) !^{2} \pi^{2}} \sum_{j_{1}=0}^{1} \sum_{j_{2}=0}^{1}(-1)^{j_{1}+j_{2}} \sum_{1 \leq m \neq n \leq z} \frac{\tau_{l}(m) \tau_{l}(n)}{(m n)^{3 / 4}} \\
& \times \cos \left(2 \pi \sqrt{m\left(x+j_{1} h\right)}-2 \pi \sqrt{n\left(x+j_{2} h\right)}\right), \\
& F_{222}(x)=\frac{2^{3-2 l} x^{2 l-1 / 2}}{(l-1) !^{2} \pi^{2}} \sum_{j_{1}=0}^{1} \sum_{j_{2}=0}^{1}(-1)^{j_{1}+j_{2}} \sum_{1 \leq m \neq n \leq z} \frac{\tau_{l}(m) \tau_{l}(n)}{(m n)^{3 / 4}} \\
& \times \sin \left(2 \pi \sqrt{m\left(x+j_{1} h\right)}+2 \pi \sqrt{n\left(x+j_{2} h\right)}\right) .
\end{aligned}
$$

Let

$$
g_{ \pm}(x)=2 \pi \sqrt{m\left(x+j_{1} h\right)} \pm 2 \pi \sqrt{n\left(x+j_{2} h\right)} .
$$

By the power series expansion

$$
(1+t)^{1 / 2}=1+\sum_{v=1}^{\infty} d_{v} t^{v} \quad(|t| \leq 1 / 2)
$$

we get that

$$
g_{ \pm}(x)=2 \pi \sqrt{x}(\sqrt{m} \pm \sqrt{n})+2 \pi \sum_{v=1}^{\infty} \frac{d_{v} h^{v}}{x^{v-1 / 2}}\left(\sqrt{m} j_{1}^{v} \pm \sqrt{n} j_{2}^{v}\right),
$$

which implies

$$
\left|g_{ \pm}^{\prime}(x)\right| \gg x^{-1 / 2}|\sqrt{m} \pm \sqrt{n}|(m \neq n)
$$

by noting that $m, n \leq \varepsilon U h^{-1}$. By the first derivative test we have

$$
\begin{aligned}
\int_{U}^{2 U} F_{221}(x) d x & \ll U^{2 l} \sum_{1 \leq m \neq n \leq z} \frac{\left|\tau_{l}(m) \tau_{l}(n)\right|}{(m n)^{3 / 4}|\sqrt{m}-\sqrt{n}|} \\
& \ll U^{2 l} \sum_{1 \leq m \neq n \leq z} \frac{d(m) d(n)}{(m n)^{3 / 4}|\sqrt{m}-\sqrt{n}|} \\
& \ll U^{2 l} \log ^{4} z,
\end{aligned}
$$

where in the last step we have used the well-known estimate

$$
\sum_{1 \leq m \neq n \leq y} \frac{d(m) d(n)}{(m n)^{3 / 4}|\sqrt{m}-\sqrt{n}|} \ll \log ^{4} y, y \geq 10 .
$$


We also have

$$
\begin{aligned}
\int_{U}^{2 U} F_{222}(x) d x & \ll U^{2 l} \sum_{1 \leq m \neq n \leq z} \frac{\left|\tau_{l}(m) \tau_{l}(n)\right|}{(m n)^{3 / 4}|\sqrt{m}+\sqrt{n}|} \\
& \ll U^{2 l} \sum_{\substack{1 \leq m<n \leq z \\
m^{3 / 4} n^{5 / 4}}} \frac{d(m) d(n)}{m^{2 l} \log ^{3} z}
\end{aligned}
$$

by the well-known estimate $\sum_{n \leq y} d(n) \ll y \log y$.

From $(5.12),(5.14)$ and $(5.15)$ we have

$$
\int_{U}^{2 U} F_{22}(x) d x \ll U^{2 l} \log ^{4} z
$$

By using the formulas

$$
\cos u-\cos v=-2 \sin \left(\frac{u+v}{2}\right) \sin \left(\frac{u-v}{2}\right)
$$

and

$$
\sin ^{2} u=(1-\cos 2 u) / 2
$$

we have

$$
\begin{gathered}
\int_{U}^{2 U} F_{21}(x) d x \\
=\frac{2^{6-2 l}}{(l-1) !^{2} \pi^{2}} \sum_{1 \leq n \leq z} \frac{\tau_{l}^{2}(n)}{n^{3 / 2}} \int_{U}^{2 U} x^{2 l-\frac{1}{2}} \sin ^{2}\left(\pi \sqrt{n(x+h)}+\pi \sqrt{n x}-\frac{\pi}{4}\right) \\
\times \sin ^{2}(\pi \sqrt{n(x+h)}-\pi \sqrt{n x}) d x=S_{1}-S_{2},
\end{gathered}
$$

for instance, where

$$
\begin{aligned}
& S_{1}=\frac{2^{5-2 l}}{(l-1) !^{2} \pi^{2}} \sum_{1 \leq n \leq z} \frac{\tau_{l}^{2}(n)}{n^{3 / 2}} \int_{U}^{2 U} x^{2 l-\frac{1}{2}} \sin ^{2}(\pi \sqrt{n(x+h)}-\pi \sqrt{n x}) d x, \\
& S_{2}=\frac{2^{5-2 l}}{(l-1) !^{2} \pi^{2}} \sum_{1 \leq n \leq z} \frac{\tau_{l}^{2}(n)}{n^{3 / 2}} \\
& \quad \times \int_{U}^{2 U} x^{2 l-\frac{1}{2}} \sin (2 \pi \sqrt{n(x+h)}+2 \pi \sqrt{n x}) \sin ^{2}(\pi \sqrt{n(x+h)}-\pi \sqrt{n x}) d x .
\end{aligned}
$$

For each $n \leq z$, let $L_{n}(t)=\int_{U}^{t} x^{2 l-1 / 2} \sin (2 \pi \sqrt{n(x+h)}+2 \pi \sqrt{n x}) d x$. By the first derivative test

$$
L_{n}(t) \ll U^{2 l} n^{-1 / 2}, U \leq t \leq 2 U \text {. }
$$


So by partial summation

$$
\begin{aligned}
& \int_{U}^{2 U} x^{2 l-\frac{1}{2}} \sin (2 \pi \sqrt{n(x+h)}+2 \pi \sqrt{n x}) \sin ^{2}(\pi \sqrt{n(x+h)}-\pi \sqrt{n x}) d x \\
& =\int_{U}^{2 U} \sin ^{2}(\pi \sqrt{n(x+h)}-\pi \sqrt{n x}) d L_{n}(x) \\
& =L_{n}(2 U) \sin ^{2}(\pi \sqrt{n(2 U+h)}-\pi \sqrt{2 n U}) \\
& -\int_{U}^{2 U} L_{n}(x) \sin (\pi \sqrt{n(x+h)}-\pi \sqrt{n x}) \cos (\pi \sqrt{n(x+h)}-\pi \sqrt{n x}) \\
& \quad \times\left(\frac{\pi \sqrt{n}}{\sqrt{x+h}}-\frac{\pi \sqrt{n}}{\sqrt{x}}\right) d x \\
& \ll U^{2 l} n^{-1 / 2}+h U^{2 l-1 / 2}
\end{aligned}
$$

Thus we get

$$
S_{2} \ll \sum_{1 \leq n \leq z} \frac{\tau_{l}^{2}(n)}{n^{3 / 2}}\left(U^{2 l} n^{-1 / 2}+h U^{2 l-1 / 2}\right) \ll U^{2 l}+h U^{2 l-1 / 2} \ll U^{2 l} .
$$

By (5.13) we have

$$
\pi \sqrt{n(x+h)}-\pi \sqrt{n x}=\frac{\pi h \sqrt{n}}{2 \sqrt{x}}+O\left(\frac{h^{2} \sqrt{n}}{x^{3 / 2}}\right),
$$

which implies that

$$
\sin ^{2}(\pi \sqrt{n(x+h)}-\pi \sqrt{n x})=\sin ^{2} \frac{\pi h \sqrt{n}}{2 \sqrt{x}}+O\left(\frac{h^{2} \sqrt{n}}{x^{3 / 2}}\right) .
$$

Thus

$$
\begin{aligned}
S_{1}= & \frac{2^{5-2 l}}{(l-1) !^{2} \pi^{2}} \sum_{1 \leq n \leq z} \frac{\tau_{l}^{2}(n)}{n^{3 / 2}} \int_{U}^{2 U} x^{2 l-\frac{1}{2}} \sin ^{2} \frac{\pi h \sqrt{n}}{2 \sqrt{x}} d x \\
& +O\left(\sum_{1 \leq n \leq z} \frac{\left|\tau_{l}^{2}(n)\right|}{n^{3 / 2}} \int_{U}^{2 U} x^{2 l-\frac{1}{2}} \frac{h^{2} \sqrt{n}}{x^{3 / 2}} d x\right) \\
= & \frac{2^{5-2 l}}{(l-1) !^{2} \pi^{2}} \sum_{1 \leq n \leq z} \frac{\tau_{l}^{2}(n)}{n^{3 / 2}} \int_{U}^{2 U} x^{2 l-\frac{1}{2}} \sin ^{2} \frac{\pi h \sqrt{n}}{2 \sqrt{x}} d x \\
& +O\left(h^{2} U^{2 l-1} \sum_{1 \leq n \leq z} \frac{\left|\tau_{l}^{2}(n)\right|}{n}\right) .
\end{aligned}
$$

Since $\left|\tau_{l}(n)\right| \leq d(n)$, we have the estimate

$$
\sum_{n \leq y} \tau_{l}^{2}(n) \ll \sum_{n \leq y} d^{2}(n) \ll y \log ^{3} y \quad(y \geq 2),
$$

which immediately implies that

$$
h^{2} U^{2 l-1} \sum_{1 \leq n \leq z} \frac{\tau_{l}^{2}(n)}{n} \ll h^{2} U^{2 l-1} \log ^{4} z \ll h U^{2 l-1 / 2} \log ^{4} U .
$$


From (5.21) we can get

$$
\begin{aligned}
& \sum_{1 \leq n \leq z} \frac{\tau_{l}^{2}(n)}{n^{3 / 2}} \int_{U}^{2 U} x^{2 l-\frac{1}{2}} \sin ^{2} \frac{\pi h \sqrt{n}}{2 \sqrt{x}} d x \\
& \ll \sum_{1 \leq n \leq z} \frac{d^{2}(n)}{n^{3 / 2}} \int_{U}^{2 U} x^{2 l-\frac{1}{2}} \min \left(1, \frac{h^{2} n}{x}\right) d x \\
& \ll h^{2} U^{2 l-1 / 2} \sum_{1 \leq n \leq U / h^{2}} \frac{d^{2}(n)}{n^{1 / 2}}+U^{2 l+1 / 2} \sum_{U / h^{2}<n \leq z} \frac{d^{2}(n)}{n^{3 / 2}} \\
& \ll h U^{2 l} \log ^{3} \frac{U}{h^{2}} \ll h U^{2 l} \log ^{3} \frac{\sqrt{U}}{h} .
\end{aligned}
$$

Combining (5.20), (5.22) and (5.23) we get

$$
S_{1} \ll h U^{2 l} \log ^{3} \frac{\sqrt{U}}{h},
$$

which together with (5.17) gives

$$
\int_{U}^{2 U} F_{21}(x) d x \ll h U^{2 l} \log ^{3} \frac{\sqrt{U}}{h} .
$$

From (5.9)-(5.11), (5.16) and (5.25) we get

$$
\int_{U}^{2 U}\left(R_{1}(x+h)-R_{1}(x)\right)^{2} d x \ll h U^{2 l} \log ^{3} \frac{\sqrt{U}}{h}+U^{2 l} \log ^{4} z .
$$

Now combining (5.5), (5.8) and (5.26) we get

$$
J(U, h) \ll h U^{2 l} \log ^{3} \frac{\sqrt{U}}{h}+U^{2 l} \log ^{4} U,
$$

which immediately implies that

$$
\int_{2} \ll h T^{2 l} \log ^{3} \frac{\sqrt{T}}{h}+T^{2 l} \log ^{4} T
$$

via a splitting argument. Finally Lemma 5.1 follows from (5.3), (5.4) and (5.27).

\section{Proof of Theorem 2}

In this section we shall prove Theorem 2. Our approach is a variant of the proof of Theorem 2 of [12].

Define

$$
R_{+}(t)= \begin{cases}R(t), & \text { if } R(t)>0 \\ 0, & \text { otherwise }\end{cases}
$$

and

$$
R_{-}(t)=|R(t)|-R_{+}(t) .
$$

We first prove the following two lemmas.

Lemma 6.1. The estimate

$$
\int_{T}^{2 T} R_{ \pm}^{2}(t) d t \gg T^{2 l+1 / 2}
$$

holds. 
Proof. From (1.11) and (1.13) with $k=4$, we get by Hölder's inequality that

$$
\begin{aligned}
T^{2 l+1 / 2} & \ll \int_{T}^{2 T} R^{2}(t) d t \leq\left(\int_{T}^{2 T}|R(t)| d t\right)^{2 / 3}\left(\int_{T}^{2 T} R^{4}(t) d t\right)^{1 / 3} \\
& \leq\left(\int_{T}^{2 T}|R(t)| d t\right)^{2 / 3} T^{4 l / 3} .
\end{aligned}
$$

Thus

$$
\int_{T}^{2 T}|R(t)| d t \gg T^{l+3 / 4} .
$$

From (3.1) and Lemma 6.1 of [32], it is easy to verify that

$$
\int_{T}^{2 T} R(t) d t \ll T^{l+1 / 2} \mathcal{L}^{2}
$$

which implies

$$
\int_{T}^{2 T} R_{ \pm}(t) d t \gg T^{l+3 / 4}
$$

in view of (6.2). By (6.3) and Cauchy-Schwarz's inequality, it follows that

$$
\begin{aligned}
T^{l+3 / 4} & \ll\left(\int_{T}^{2 T} d t\right)^{1 / 2}\left(\int_{T}^{2 T} R_{ \pm}^{2}(t) d t\right)^{1 / 2} \\
& \ll T^{1 / 2}\left(\int_{T}^{2 T} R_{ \pm}^{2}(t) d t\right)^{1 / 2}
\end{aligned}
$$

which immediately implies Lemma 6.1.

Lemma 6.2. Suppose $2 \leq H \leq \sqrt{T}$. Then

$$
\int_{T}^{2 T} \max _{h \leq H}\left(R_{ \pm}(t+h)-R_{ \pm}(t)\right)^{2} d t \ll H T^{2 l} \log ^{5} T .
$$

Proof. It is easy to verify that

$$
\left|R_{ \pm}(t+h)-R_{ \pm}(t)\right| \leq|R(t+h)-R(t)|,
$$

so it is sufficient to prove the estimate

$$
I=\int_{T}^{2 T} \max _{h \leq H}(R(t+h)-R(t))^{2} d t \ll H T^{2 l} \log ^{5} T .
$$

Write

$$
H=2^{\lambda} b
$$


such that $\lambda \in \mathbb{N}$ and $1 \leq b<2$. Similar to the argument of the proof of Lemma 2 of [12], we can deduce by using Lemma 5.1 that

$$
\begin{aligned}
I & \ll \lambda \sum_{\mu \leq \lambda} \sum_{0 \leq \nu<2^{\mu}} \int_{T+\nu 2^{\lambda-\mu} b}^{2 T+\nu 2^{\lambda-\mu} b}\left|R\left(t+2^{\lambda-\mu} b\right)-R(t)\right|^{2} d t+T^{2 l} \log ^{2} T \\
& \ll \lambda \sum_{\mu \leq \lambda} \sum_{0 \leq \nu<2^{\mu}}\left(T^{2 l} 2^{\lambda-\mu} b \log ^{3} T+T^{2 l} \log ^{4} T\right) \\
& \ll \lambda \sum_{\mu \leq \lambda}\left(T^{2 l} 2^{\lambda} b \log ^{3} T+2^{\mu} T^{2 l} \log ^{4} T\right) \\
& \ll \lambda^{2} H T^{2 l} \log ^{3} T+\lambda H T^{2 l} \log ^{4} T \\
& \ll H T^{2 l} \log ^{5} T
\end{aligned}
$$

namely (6.4) holds.

Now we finish the proof of Theorem 2. For any function $P(t)$ and $Q(t)$, if

$$
\omega(t)=P^{2}(t)-4 \max _{h \leq H}(P(t+h)-P(t))^{2}-Q^{2}(t)>0,
$$

then

$$
|P(t)| \geq 2 \max _{h \leq H}|P(t+h)-P(t)|
$$

and

$$
|P(t)| \geq|Q(t)|
$$

The first inequality implies, for any $0 \leq h \leq H$,

$$
P(t)-\frac{1}{2}|P(t)| \leq P(t+h) \leq P(t)+\frac{1}{2}|P(t)|
$$

and hence $P(t+h)$ has the same sign as $P(t)$. Moreover, by the second inequality above we get

$$
|P(t+h)| \geq \frac{1}{2}|P(t)| \geq \frac{1}{2}|Q(t)| .
$$

Now take $P(t)=R_{ \pm}(t)$ and $Q(t)=\delta t^{l-1 / 4}$ for a sufficiently small $\delta>0$. By Lemma 6.1 and Lemma 6.2 we get

$$
\begin{aligned}
\int_{T}^{2 T} \omega(t) d t & \gg T^{2 l+1 / 2}-O\left(H T^{2 l} \log ^{5} T\right)-O\left(\delta^{2} T^{2 l+1 / 2}\right) \\
& \gg T^{2 l+1 / 2}
\end{aligned}
$$

by taking $H=\delta \sqrt{T} \log ^{-5} T$. Let $\mathscr{S}=\{t \in[T, 2 T]: \omega(t)>0\}$. By (6.5), the Cauchy-Schwarz inequality and (1.13) with $k=4$ we get

$$
\begin{aligned}
T^{2 l+1 / 2} & \ll \int_{T}^{2 T} \omega(t) d t \leq \int_{\mathscr{S}} \omega(t) d t \leq \int_{\mathscr{S}} R_{ \pm}^{2}(t) d t \\
& \leq|\mathscr{S}|^{1 / 2}\left(\int_{T}^{2 T} R^{4}(t) d t\right)^{1 / 2} \ll|\mathscr{S}|^{1 / 2} T^{2 l} .
\end{aligned}
$$

Thus we get

$$
|\mathscr{S}| \gg T \text {. }
$$

This completes the proof of Theorem 2 . 
Remark for Theorem 4 . The proof of Theorem 4 is the same except that we use $\log ^{3} T$ instead of $\log ^{5} T$. Here $\log ^{3} T$ appears since for $R_{I}(t)$ we can prove that the estimate

$$
\int_{1}^{T}\left(R_{I}(t+h)-R_{I}(t)\right)^{2} d t \ll h T \log \frac{\sqrt{T}}{h}+T \mathcal{L} \log \mathcal{L}
$$

holds for $1 \leq h \leq \sqrt{T} / 2$, which implies that the $\log ^{5} T$ in Lemma 6.2 can be replaced by $\log ^{3} T$ if we have $R_{I}(t)$ in place of $R(t)$.

\section{Proof of Theorem 3}

In this section we prove Theorem 3 . Suppose $k \geq 3$ is a fixed odd integer and $T$ is a large parameter. Define

$$
\delta= \begin{cases}-1, & \text { if } C_{k, l} \geq 0 \\ 1, & \text { if } C_{k, l}<0,\end{cases}
$$

where $C_{k, l}$ is defined in the formula (1.13).

By Theorem 2, let $t \in[T, 2 T]$ such that

$$
\delta R(u)>c_{5} t^{l-1 / 4}, u \in[t, t+H],
$$

with $H=c_{4} \sqrt{T} \log ^{-5} T$. Then

$$
\begin{aligned}
c_{5}^{k} H t^{k(l-1 / 4)}< & \int_{t}^{t+H} \delta^{k} R^{k}(u) d u=\delta^{k} \int_{t}^{t+H} R^{k}(u) d u \\
= & C_{k, l} \delta^{k}\left((t+H)^{k(l-1 / 4)+1}-t^{k(l-1 / 4)+1}\right) \\
& +\delta^{k}\left(\mathcal{F}_{k, l}(t+H)-\mathcal{F}_{k, l}(t)\right) \\
= & C_{k, l} \delta^{k}(k(l-1 / 4)+1) H t^{k(l-1 / 4)}+O\left(H^{2} t^{k(l-1 / 4)-1}\right) \\
& +\delta^{k}\left(\mathcal{F}_{k, l}(t+H)-\mathcal{F}_{k, l}(t)\right) .
\end{aligned}
$$

Hence we get

$$
\delta^{k}\left(\mathcal{F}_{k, l}(t)-\mathcal{F}_{k, l}(t+H)\right)<B_{k, l} H t^{k(l-1 / 4)}\left(1+O\left(H T^{-1}\right)\right),
$$

where

$$
B_{k, l}=C_{k, l} \delta^{k}(k(l-1 / 4)+1)-c_{5}^{k} \leq-c_{5}^{k}<0 .
$$

From (7.1) and (7.2) we have

$$
\left|\mathcal{F}_{k, l}(t)-\mathcal{F}_{k, l}(t+H)\right| \gg H t^{k(l-1 / 4)},
$$

and Theorem 3 hence follows.

\section{REFERENCES}

[1] V. Bentkus and F. Götze, Lattice point problems and distribution of values of quadratic forms, Ann. of Math. (2) 50:3(1999), 977-1027. MR.1740988(2001b:11087)

[2] P. H. Bérard, On the wave equation on a compact Riemannian manifold without conjugate points, Math. Z. 155:3(1977), 249-276. MR0455055 (56:13295)

[3] L. Bleher, On the distribution of the number of lattice points inside a family of convex ovals, Duke Math. J. 67:3(1992), 461-481. MR.1181309 (93h:11110)

[4] D. Chung, Y. N. Petridis and J. Toth, The remainder in Weyl's law for Heisenberg manifolds II, Bonner Mathematische Schriften, Nr. 360, Bonn, 2003, 16 pages. MR.2075620 (2005i:58035) 
[5] H. Cramér, Über zwei Sätze von Herrn G. H. Hardy, Math. Z. 15(1922), 201-210. MR 1544568

[6] G. B. Folland, Harmonic Analysis in Phase Space, Princeton University Press (1989), 9-73. MR.983366 (92k:22017)

[7] F. Fricker, Einführung in die Gitterpunketlehre, [Introduction to lattice point theory] Lehrbücher und Monographien aus dem Gebiete der Exakten Wissenschaften (LMW), Mathematische Reihe [Textbooks and Monographs in the Exact Sciences] 73, Birkhäuser Verlag, Basel-Boston, 1982. MR673938 (84b:10001)

[8] A. Good, Ein $\Omega$-Resultat für das quadratische Mittel del Riemannschen Zetafunktion auf der kritische Linie, Invent. Math. 41(1977), 233-251. MR0460253 (57:247)

[9] C. Gordon and E. Wilson, The spectrum of the Laplacian on Riemannian Heisenberg manifolds, Michigan Math. J. 33:2(1986), 253-271. MR837583 (87k:58275)

[10] F. Götze, Lattice point problems and values of quadratic forms, Inventiones Mathematicae 157(2004), 195-226. MR2135188 (2005m:11185)

[11] G. H. Hardy, On the expression of a number as the sum of two squares, Quart. J Math. 46(1915), 263-283.

[12] D. R. Heath-Brown and K.Tsang, Sign changes of $E(t), \Delta(x)$ and $P(x)$, J. of Number Theory 49(1994), 73-83. MR 1295953 (96a:11093)

[13] L. Hörmander, The spectral function of an elliptic operator, Acta Math. 121(1968), 193-218. MR0609014 (58:29418)

[14] M. N. Huxley, Exponential sums and lattice points III, Proc. London Math. Soc. 87:3(2003), 591-609. MR2005876 (2004m:11127)

[15] A. Ivić, The Riemann-zeta function, John Wiley \& Sons, New York, 1985. MR792089 (87d:11062)

[16] A. Ivić and Wenguang Zhai, Higher moments of the error term in the divisor problem, to appear in Mat. Zametki.

[17] V. YA. Ivrii, Precise Spectral Asymptotics for Elliptic Operators Acting in Fibrings over Manifolds with Boundary, Springer Lecture Notes in Mathematics 1100(1984). MR771297 (86h:58139)

[18] M. Jutila, On the divisor problem for short intervals, Ann. Univ. Turkuensis Ser. AI 186(1984), 23-30. MR748516 (85i:11077)

[19] M. Khosravi, Third moment of the remainder error term in Weyl's law for Heisenberg manifolds, arXiv: 0711.0073.

[20] M. Khosravi and Y. Petridis, The remainder in Weyl's law for $n$-dimensional Heisenberg manifolds, Proc. of the American Math. Soc. 133(2005), 3561-3571. MR 2163591(2006e:58045)

[21] M. Khosravi and J. Toth, Cramér's formula for Heisenberg manifolds, Ann. de l'institut Fourier 55(2005), 2489-2520. MR2207391 (2007b:58043)

[22] Y. Lau and K. Tsang, On the mean square formula of the error term in the Dirichlet divisor problem, Math. Proc. Camb. Phil. Soc. 146(2009), 277-287. MR2475967(2009k:11149)

[23] W. G. Nowak, A lower bound for the error term in Weyl's law for certain Heisenberg manifolds, arXiv: 0809.3924.

[24] W. G. Nowak, A lower bound for the error term in Weyl's law for certain Heisenberg manifolds.II, arXiv: 0810.2235 .

[25] Y. Petribis and J. Toth, The remainder in Weyl's law for Heisenberg manifolds, J. Differential Geom. 60(2002), 455-483. MR1950173(2004c:58054)

[26] K. Soundararajan, Omega results for the divisor and circle problems, J. Int. Math. Res. Not. 36(2003), 1987-1998. MR1991181 (2004f:11105)

[27] E. M. Stein, Harmonic Analysis, Princeston University Press (1993), 527-574. MR 1232192 (95c:42002)

[28] Kai-Man Tsang, Higher-power moments of $\Delta(x), E(t)$ and $P(x)$, Proc. London Math. Soc. 65:3(1992), 65-84. MR:1162488 (93c:11082)

[29] A. V. Volovoy, Improved two-term asymptotics for the eigenvalue distribution function of an elliptic operator on a compact manifold, Comm. Partial Differential Equations 15:11(1990), 1509-1563. MR 1079602 (91m:58158)

[30] Wenguang Zhai, On higher-power moments of $\Delta(x)$ (II), Acta Arith. 114(2004), 35-54. MR2067871 (2005h:11216)

[31] Wenguang Zhai, On higher-power moments of $\Delta(x)$ (III), Acta Arith. 118(2005), 263-281. MR2168766 (2006f:11121) 
[32] Wenguang Zhai, On the error term in the Weyl's law for Heisenberg manifolds, Acta Arith. 134(2008), 219-257. MR2438847 (2009h:11154)

Department of Mathematics, University of Hong Kong, Pokfulam road, Hong Kong

E-mail address: kmtsang@maths.hku.hk

Department of Mathematics, China University of Mining and Technology, Beijing 100083, People's Republic of China

E-mail address: zhaiwg@hotmail.com 